\title{
Ações do Grupo de Apoio Pedagógico (GAP) em Prol do Ensino de Graduação da Faculdade de Zootecnia e Engenharia de Alimentos (FZEA)
}

\author{
Lia de Alencar Coelho*1, Marcelo Machado De Luca de Oliveira Ribeiro ${ }^{1}$, Valdo Rodrigues
} Herling ${ }^{2}$, Helena Lage Ferreira ${ }^{3}$, Samantha Cristina de Pinho ${ }^{4}$, Rubens André Tabile ${ }^{5}$, Carmo Gabriel da Silva Filho ${ }^{6}$, Gelson José Andrade da Conceição ${ }^{1}$, Giovana Tommaso ${ }^{1}$, Ana Maria Centola Vidal Martins ${ }^{3}$, Marta Mitsui Kushida ${ }^{4}$, Ana Carolina de Souza Silva ${ }^{5}$

${ }^{1}$ Comissão de Graduação da Faculdade de Zootecnia e Engenharia de Alimentos da Universidade de São Paulo ${ }^{2}$ Comissão Coordenadora de Curso $(\mathrm{CoC})$ da Zootecnia

${ }^{3} \mathrm{CoC}$ Medicina Veterinária

${ }^{4} \mathrm{CoC}$ Engenharia de Alimentos

${ }^{5} \mathrm{CoC}$ Engenharia de Biossistemas

${ }^{6}$ Estudante de Graduação da Faculdade de Zootecnia e Engenharia de Alimentos

* Autor para correspondência:

\section{RESUMO}

O presente relato apresenta as ações exercidas pelo GAP-FZEA durante o período de 2005 a 2016 e o seu impacto nas atividades desenvolvidas pelos docentes da FZEA pertinentes ao ensino de graduação. Antes da criação das Comissões Coordenadoras de Curso (CoCs), o GAP-FZEA atuou diretamente na promoção de eventos sobre gestão pedagógica dando subsídios aos docentes para que os mesmos refletissem sobre a necessidade de traçar o perfil profissional do estudante e sobre sua própria formação pedagógica. Após a criação das CoCs, o GAPFZEA, além de continuar trabalhando na formação pedagógica dos docentes da FZEA, atuou indiretamente na gestão pedagógica dando subsídios às CoCs da FZEA para que as mesmas reflitam sobre a construção dos Projetos Políticos Pedagógicos (PPPs). Posteriormente, o GAP-FZEA promoveu o I Simpósio de Graduação da FZEA, que permitiu a troca de experiências vivenciadas pelos docentes da FZEA quanto à gestão e à prática pedagógica. O GAP-FZEA mostrou que pode propiciar espaços para congregar os docentes e estimular o debate sobre as práticas de ensino-aprendizagem na construção desse ambiente de aprimoramento.

Palavras-Chave: Coordenação de Cursos; Desenvolvimento Docente; Projeto Político Pedagógico.

\begin{abstract}
This report presents the activities performed by GAP-FZEA during the period of 2005 to 2016 and their impact on the occupation of FZEA teachers in relation to teaching/learning process. Before the creation of Course Coordinating Committees (CoCs), the GAP-FZEA helped the FZEA teachers to draw the professional profile of Higher Education students and also helped them to think about their own pedagogical formation. After the CoCs creation, besides the GAP-FZEA was continuing to work in direction to pedagogical formation of the teachers, they mainly focus on helping the CoCs of FZEA in thinking about the construction of PoliticalPedagogical Project (PPP). Later, the GAP-FZEA promoted the I Simpósio de Graduação da FZEA, which give to teachers the opportunity to exchange experiences regarding to pedagogical management and practice. The GAP-FZEA provides favorable conditions to congregate the teachers by stimulating the discussion about teaching/learning process.
\end{abstract}

Keywords: Course Coordinating; Teacher Development; Political Pedagogic Project.

\section{Introdução}

O Grupo de Apoio Pedagógico da FZEA (GAP-FZEA), criado a partir da Portaria Interna da
Pró-Reitoria de Graduação da USP (n. 04/2004), tem a finalidade de dar subsídios à Comissão de Graduação (CG), às Comissões Coordenadoras de 
Cursos (CoCs) e aos docentes da FZEA para que renovem e aprofundem os conhecimentos necessários para promover mudanças quanto à gestão e à prática pedagógica. Atualmente o GAP-FZEA é composto por quatro docentes e os respectivos suplentes, sendo um representante de cada curso da FZEA, indicados por cada CoC, dois docentes e os respectivos suplentes, recomendados pela CG, e um representante discente da graduação e respectivo suplente, escolhidos pelo Centro Acadêmico da FZEA. Em época anterior, a gestão pedagógica se dava no âmbito do Departamento e da Comissão de Graduação, existindo apenas a "figura" do Coordenador de Curso. Essa disposição não permitia que os docentes de um dado curso refletissem em conjunto sobre o ensino de graduação. A partir da criação das CoCs mediante a resolução do Conselho de Graduação (CoG) n. 5500, de 13 de janeiro de 2009 e da resolução CoG, n. 5100, de 11 de fevereiro de 2009, que baixou o regimento da Comissão de Apoio Pedagógico (CAP), foi possível a institucionalização de espaços específicos de aperfeiçoamento docente considerando a gestão e a prática pedagógica (CORRÊA et al., 2011), visto que a busca de conhecimentos sobre o desenvolvimento docente tem sido uma temática estudada por diversos autores (NÓVOA, 1992; VEIGA, 2008; PEREIRA \& BEHRENS, 2010). Neste relato serão expostas três etapas que envolvem as ações promovidas pelo GAP-FZEA antes (primeira etapa) e após (segunda etapa) a criação das CoCs e as atividades dos docentes da FZEA centradas na gestão e prática pedagógica diante dos conhecimentos adquiridos durante os eventos promovidos pelo GAP-FZEA (terceira etapa).

\section{Ações do GAP-FZEA antes da Criação das CoCs}

Na primeira etapa, as ações do GAP-FZEA se concentraram na promoção de eventos que propiciassem aos docentes a renovação e o aprofundamento dos conhecimentos necessários à gestão pedagógica, mas poucos eventos destinados ao desenvolvimento docente foram realizados.

Considerando a Gestão Pedagógica, o GAP-FZEA desenvolveu uma programação articulada de três encontros (workshops) sequenciados, com a finalidade de incitar os docentes a refletirem sobre o ensino de graduação quanto a sua organização. O primeiro workshop, além de contar com a participação de um palestrante externo, foi dividido em três períodos: manhã (1), tarde (1) e manhã (2). No período da tarde, os docentes se reuniram em subgrupos, conforme as áreas do conhecimento dentro de cada curso. A formação dos subgrupos teve as seguintes finalidades: a) reflexão sobre a grade curricular de cada curso; b) integração entre docentes responsáveis por disciplinas inseridas dentro de uma mesma área de conhecimento; c) contribuição para o desenvolvimento do perfil profissional desejado. Cada subgrupo recebeu uma pasta contendo: 1) Estrutura Curricular por Curso (disciplinas e respectivos pré-requisitos distribuídos por semestre); 2) Conteúdo Programático de cada Disciplina do Curso; 3) Lista com as modificações realizadas na grade curricular para o ano seguinte; 4) Questionário com três perguntas envolvendo a qualidade de ensino sob o aspecto da articulação com outros docentes/disciplinas e o da "repetição" de conteúdo programático. $\mathrm{Na}$ manhã 2 , houve a conclusão da discussão setorial (subgrupos) com a apresentação da síntese da reunião de cada subgrupo por um de seus membros (relator) e mesa -redonda constituída pela diretoria, coordenadores de curso e membros do GAP-FZEA. O segundo workshop contou com a participação de um palestrante externo (manhã), e a formação dos subgrupos (tarde) foi mantida. Entretanto, como não foi possível essa dinâmica para o Curso de Zootecnia (ZOO) no evento anterior, essa formação e os procedimentos durante a reunião setorial se mantiveram os mesmos, tal como ocorreu no primeiro evento. Para o curso de Engenharia de Alimentos (EA), houve recombinação dos subgrupos considerando os pré-requisitos das disciplinas e seus respectivos responsáveis. Nesse caso, os subgrupos recombinados responderam outro questionário envolvendo a qualidade do ensino sob o aspecto de produção de conhecimento, básico e aplicado e grau de importância das disciplinas. A conclusão da reunião setorial do segundo evento foi realiza- 
da no terceiro workshop, no dia 7 de novembro de 2005. Dessa forma, esse evento se restringiu apenas à apresentação dos resultados obtidos nos dois primeiros eventos. Alguns tópicos foram levantados a partir dos resultados obtidos com a formação dos subgrupos por área do conhecimento dentro de cada curso, Engenharia de Alimentos e Zootecnia. Em função do número de participantes (Figura 1), só foi possível a formação dos subgrupos no curso de EA, entretanto os docentes presentes do curso $\mathrm{ZOO}$ formaram um único grupo e discutiram alguns pontos gerais pertinentes ao curso. Para o curso EA, a formação dos subgrupos possibilitou o conhecimento da Estrutura Curricular do Curso pelos docentes recém-contratados; foram detectados alguns pontos que envolvem os pré-requisitos de disciplinas e repetição de conteúdos programáticos; verificou-se necessária a definição do perfil profissional desejado. Para o curso ZOO, o grupo considerou que havia necessidade de formação dos subgrupos; que, embora o curso estivesse consolidado e a repetição de conteúdos programáticos já tivesse sido discutida anteriormente, ainda requeria reajustes; também havia necessidade de definir o perfil profissional desejado; a reflexão sobre o ensino da ZOO devia acontecer de forma mais abrangente, sob gerência institucional.

O sucesso da formação dos subgrupos no curso EA foi atribuído à expressiva participação dos docentes do Departamento de Engenharia de Alimentos (ZEA) que, na época (2005), era composto basicamente por professores recém-contratados. De fato, o percentual médio de participação dos docentes por departamento variou em função do tempo de serviço. O nível de participação dos docentes com até seis anos de USP foi maior no ZEA e no Departamento de Ciências Básicas (ZAB), composto por docentes que são responsáveis pela escolaridade de disciplinas de ambos os cursos. Para o curso ZOO, a formação dos subgrupos pareceu não ser o método de trabalho mais adequado e isso provavelmente em função de este curso já estar estruturalmente consolidado, sendo considerado um dos cinco melhores cursos de zootecnia do país pelo Guia do Estudante, elaborado pela editora Abril. Em ambos os cursos, o apoio institucional pareceu ser importante. No ZEA, a participação docente foi maciça no primeiro evento e menor no segundo (Figura 1). Isso provavelmente se deve ao reduzido envolvimento de docentes representantes dos Órgãos Institucionais no segundo evento (Figura 2).

Considerando o desenvolvimento docente, o GAP-FZEA promoveu dois eventos envolvendo Metodologias de Ensino (MEs) e um evento abrangendo as Tecnologias de Informação e Comunicação (TICs) com o treinamento na plataforma COL (Cursos On Line) mantido em colaboração pela Coordenadoria de Tecnologia da Informação (CTI), pelo Centro de Computação Eletrônica (CGE) e pelo Laboratório de Arquitetura e Redes de Computadores (LARG) da Escola Politécnica da USP. Quanto às MEs, além da palestra sobre "Ensino-Aprendizagem no Ensino Superior" ministrada por um docente da Faculdade de Educação da Unicamp, foi realizado um curso de três dias ministrado por um docente da EESC/USP. O primeiro dia do curso constou de miniaulas dos próprios participantes, de quinze minutos, enfocando um tópico das suas disciplinas de graduação. No segundo dia, o especialista fez sua exposição adaptando o seu conteúdo programático em função do que foi exposto nas miniaulas. No terceiro dia, procedeu a um atendimento individual com cada um dos participantes, vinte minutos para cada um. Houve uma discussão comparativa entre as miniaulas e a exposição realizada no segundo dia.

\section{Ações do GAP-FZEA após a Criação das CoCs}

Na segunda etapa, a FZEA já contava com a criação de dois novos cursos: Engenharia de Biossistemas (EB) e Medicina Veterinária (MV). Dentro desse contexto, as ações do GAP-FZEA ainda se concentraram na promoção de eventos que tratavam da gestão pedagógica, mas destinados às CoCs para que seus membros pudessem renovar e aprofundar os conhecimentos necessários à gestão pedagógica. Na primeira etapa, quando da formação dos subgrupos, os docentes dos cursos EA e ZOO já haviam pontuado sobre a necessidade de 


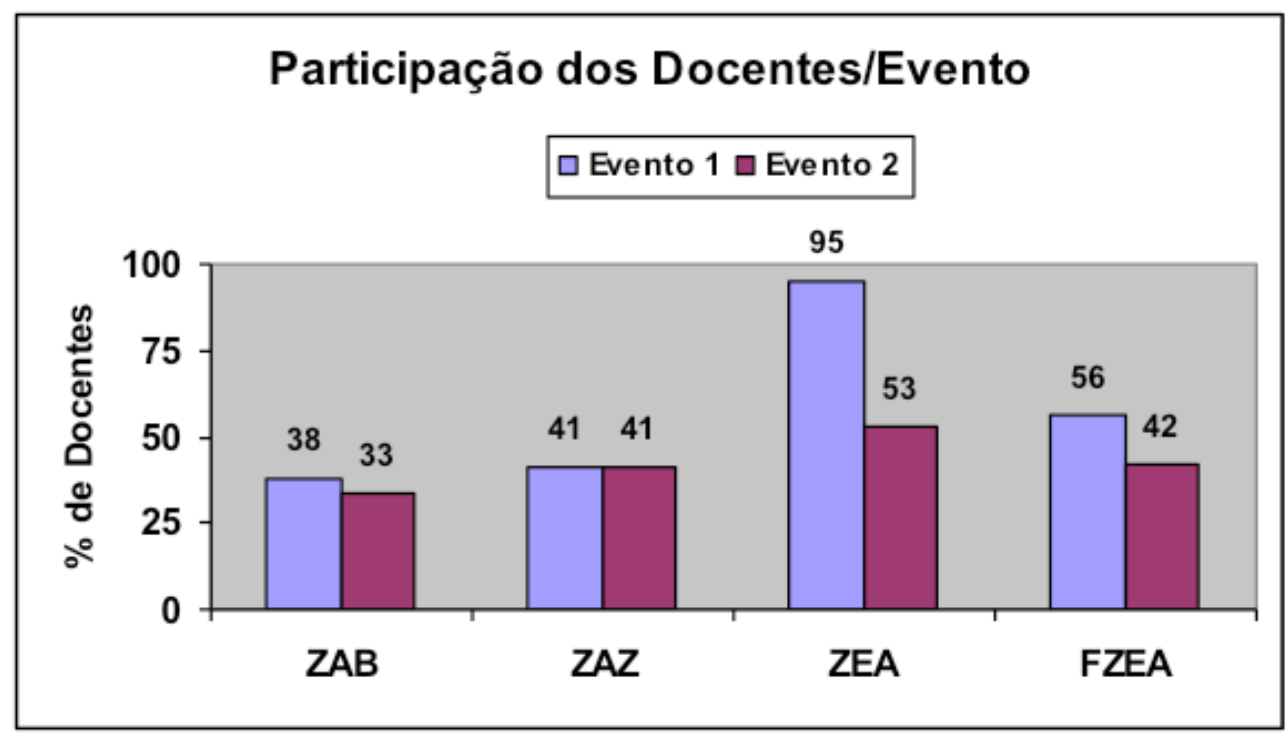

Figura 1 - Participação (\%) dos docentes dos Departamentos de: Ciências Básicas (ZAB), Zootecnia (ZAZ) e Engenharia de Alimentos (ZEA) da FZEA no I (Evento 1) e II (Evento 2) workshops do GAP-FZEA.

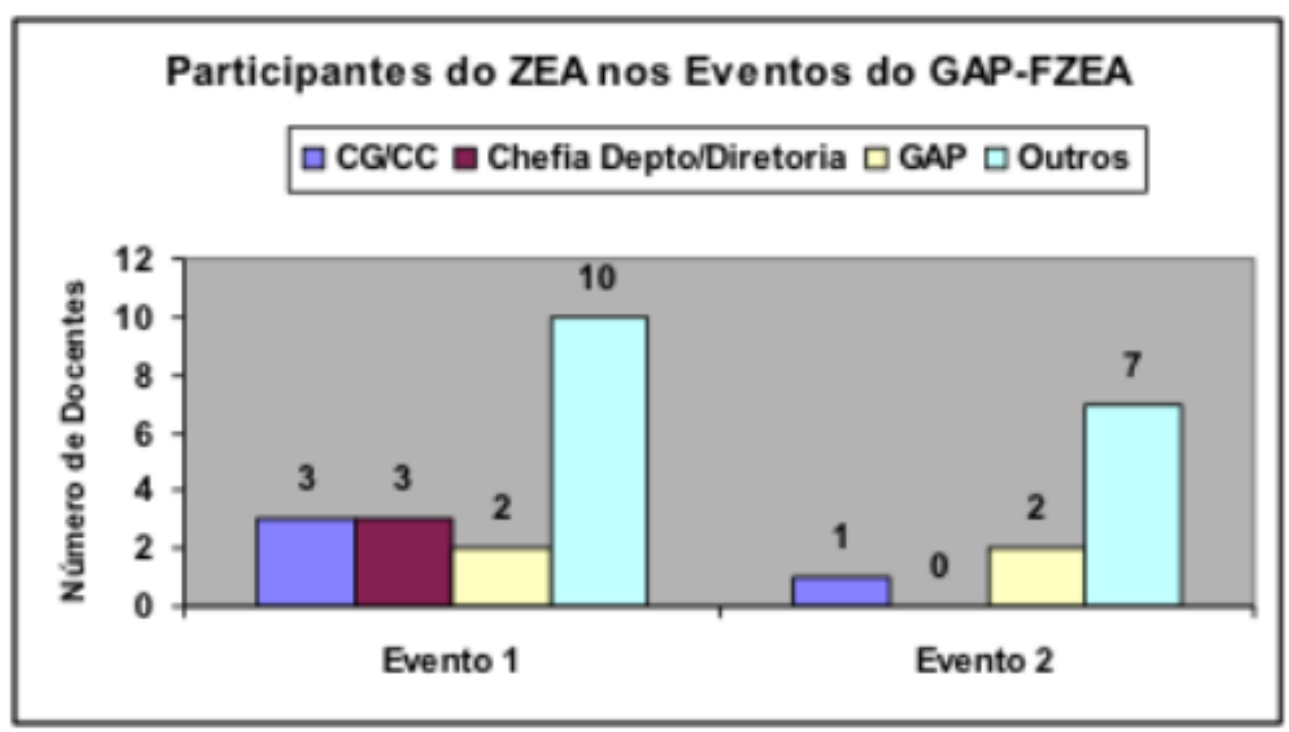

Figura 2 - Número de docentes do ZEA representantes de órgãos institucionais ou que participaram no I (Evento 1) e II (Evento 2) workshops do GAP-FZEA. CG = Comissão de Graduação. $\mathrm{CC}=$ Coordenador de Curso.

definição do perfil profissional desejado. Nesse sentido, o GAP-FZEA concentrou as suas ações em promover eventos que propiciassem o treinamento das CoCs dos Cursos EA, ZOO, MV e EB para que as mesmas reestruturassem os Projetos Políticos Pedagógicos (PPPs) e os respectivos currículos. Entretanto, como a construção curricular constitui um processo coletivo (VEIGA et al., 2013, p. 99), foi permitida a participação dos demais docentes e também dos estudantes de graduação e pós-graduação. Realizou-se uma sequência de duas oficinas e um workshop sobre PPP.
A primeira oficina, ministrada por docentes do Campus de Ribeirão Preto (EERP e FFGLRP), forneceu subsídios aos participantes para que os mesmos pudessem perceber que a construção do PPP é um processo que envolve a coletividade; tem que estar articulado com currículo e com as Diretrizes Curriculares Nacionais para os cursos de graduação; e que sua construção não se limita à redução de carga horária e à abolição de pré-requisitos. Também ficou claro que o Apoio Institucional é muito relevante, principalmente para estimular a participação do corpo docente. 
A segunda oficina contou com a participação de docentes da EE/USP, além dos professores do Campus de Ribeirão Preto, presentes na primeira oficina. $\mathrm{O}$ workshop sobre PPP, que também teve a participação dos docentes externos antes mencionados, foi estruturado da seguinte forma: as CoCs da FZEA apresentaram propostas de mudanças da estrutura curricular e dos respectivos PPPs a partir das atividades práticas desenvolvidas durante as oficinas. As professoras convidadas apresentaram sugestões para melhoria de tais propostas. Esse evento foi extremamente bem-sucedido: contou com expressiva participação de docentes de todos os departamentos e/ou cursos da FZEA, inclusive com a presença do diretor da FZEA.

Como o espaço de discussão, criado em torno dos PPPs, permitiu a elaboração de diversos questionamentos em torno das práticas de ensino -aprendizagem dos docentes, durante esse período, o GAP-FZEA também promoveu eventos ligados ao desenvolvimento docente, que estão expostos no Quadro 1. Todos têm possibilitado que os docentes mais novos (contratados em função da criação dos Cursos MV e EB) e também os mais antigos desenvolvam atividades em prol da melhoria do ensino de graduação.

\section{Atividades dos Docentes da FZEA diante dos Conhecimentos Adquiridos durante os Eventos Promovidos pelo GAP-FZEA}

Após diversas atividades com o objetivo de promover o desenvolvimento docente quanto à gestão e à prática pedagógica, o GAP-FZEA realizou uma consulta aos professores da FZEA para conhecer como tem sido seu desempenho em termos de metodologias de ensino, avaliação da aprendizagem e utilização de programas institucionais voltados para a melhoria do ensino de graduação. Também foi feita uma consulta sobre as percepções dos docentes da FZEA em relação à respectiva formação pedagógica para o desenvolvimento docente (COELHO et al., 2016). A partir dessa enquete, foi possível avaliar o impacto das ações GAP-FZEA no desenvolvimento docente no âmbito da Unidade. Partindo dessa premissa, o
GAP-FZEA promoveu o I Simpósio de Graduação da FZEA, em setembro de 2016. O simpósio visou à integração de professores, pós-doutorandos e estudantes de pós-graduação e graduação mediante o compartilhamento de experiências relacionadas à gestão e a práticas pedagógicas aplicadas no ensino de graduação. O GAP-FZEA também utilizou o simpósio para constituir um espaço importante para que os estudantes de graduação apresentassem suas práticas nas atividades complementares em sua formação, quer sejam desenvolvidas junto às Empresas Juniores, Grupos de Estudos, Programas de Tutoria ou em outras agremiações. As palestras, as apresentações orais, os resumos simples e os resumos expandidos abordaram três temas: (1) Ensino-Aprendizagem, (2) Gestão Pedagógica e (3) Atividades de Formação Complementar dos Estudantes de Graduação. Das sete apresentações orais, três abordavam o tema 1 e quatro o tema 2 (apresentação das CoCs). Dos 37 resumos apresentados como pôster, quinze, treze e nove abordavam os temas 1,2 e 3 , respectivamente. $\mathrm{O}$ simpósio gerou dezoito resumos expandidos e 22 resumos simples, que estão publicados nos Anais sob a forma de e-book (ISBN 978-85-66404-08-1) e alojados no site da FZEA (http://www.fzea.usp. br/Ppage_id=16145). A realização do simpósio possibilitou a divulgação dos trabalhos desenvolvidos pelos docentes e estudantes de graduação da FZEA em prol do ensino de graduação.

\section{Conclusão}

A formação pedagógica do professor para a educação superior tem se constituído um desafio para as Instituições de Educação Superior, tendo em vista que a docência se faz por uma ação prática, dinâmica, carregada de sentido e ambígua, na qual não está em si assegurado o seu êxito. Ficou claro que o GAP-FZEA se tornou um promotor importante de avanços na formação pedagógica complementar dos docentes da Unidade. O oferecimento dos cursos e eventos coordenados pelo Grupo tem respondido, em parte, às demandas apresentadas pelos docentes relacionadas à prática e à gestão pedagógica. Quando se observam os 


\begin{tabular}{|c|c|c|c|}
\hline & \multicolumn{2}{|c|}{ Temas } & \\
\hline Ano & MEs & Total \\
\hline 2010 & 1 & 1 & \\
\hline 2011 & 2 & 0 & 2 \\
\hline 2012 & 0 & 2 & 2 \\
\hline 2013 & 1 & 2 & 3 \\
\hline 2014 & 1 & 0 & 1 \\
\hline 2015 & 1 & 1 & 2 \\
\hline
\end{tabular}

Quadro 1 - Número de Eventos Promovidos pelo GAP-FZEA Abordando os Temas Metodologias de Ensino (MEs) e Tecnologias da Informação e Comunicação (TICs).

processos de elaboração dessas práticas no contexto da formação pedagógica dos docentes, aparece a necessidade de constante e crescente atualização, de forma que permita criar ambientes favoráveis à melhoria e à valorização do ensino de graduação na FZEA. O GAP-FZEA pode propiciar espaços para congregar os docentes e estimular o debate sobre as práticas de ensino-aprendizagem na construção desse ambiente de aprimoramento. Também ficou claro que o apoio institucional é um fator importante para o sucesso das ações promovidas pelo GAP-FZEA em prol do ensino de graduação.

\section{Referências Bibliográficas}

COELHO, L. de A.; RIBEIRO, M. M. L. O.; HERLING, V. R.; PINHO, S. C.; KUSHIDA, M. M.; KEID, L. B.; HAGE, M. C. F. N. S.; FERREIRA, H. L.; MARTINS, A. M. C. V; TABILE, R. A. ; SILVA, A. G. S; CONCEIÇÃO, G. J. A.; TOMMASO, G.; SILVA FILHO, C. G. \& OLIVEIRA, B. D. "Caracterização das Atividades Ligadas ao Ensino de Graduação dos Docentes da FZEA". Anais do I Simpósio de Graduação da FZEA, Pirassununga-SP, set. 2016, pp. 94-100. Disponível em: 〈http://www.fzea.usp.br/?page_id=16145>. Acessado em 15 nov. 2017.
CORREA, A. K.; BÓGUS, C. M.; ANASTASIOU, L. G. C.; COELHO, L. de A.; DANTAS, L. E. P. B. T.; RIVAS, N. P. P.; LIGUORI NETO, R.; JOÃO, S. M. A.; HAGE, S. R. V.; PUSCHEL, V. A. A. \& IAMAMOTO, Y. "Formação Pedagógica do Professor Universitário: Reflexões a Partir de uma Experiência". In: PIMENTA, S. G. \& ALMEIDA, M. I. (orgs.). Pedagogia Universitária: Caminhos para Formação de Professores. São Paulo: Cortez, 2011, vol.1, pp. 75-100.

NÓVOA, A. "Formação de Professores e Profissão Docente". In: ção. Lisboa: Dom Quixote, 1992.

PEREIRA, L. \& BEHRENS, M. A. "Desenvolvimento Docente no Ensino Superior: Visibilidade e Atuação Profissional". Revista Práxis Educativa, vol. 5, n. 1, pp. 39-46, 2010. ISSN 1809-4031. Disponível em: $<$ http://www.uacm.kirj.redalyc.redalyc.org/articulo. oa?id=89413516004> $>$. Acessado em 15 nov. 2017.

VEIGA, I. P. A. "Docência como Atividade Profissional". In: VEIGA, I. P. A \& ÁVILA, C. (orgs.). Profissão Docente: Novos Sentidos, Novas Perspectivas. Campinas: Papirus, 2008.

; SOUZA, M. H. V. \& GABIN, N. Desenvolvimento Profissional Docente: Currículo, Docência e Avaliação na Educação Superior. Recife: Universitária UFPE, 2013, vol. $1.240 \mathrm{p}$.

Publicado em 22/12/2017. 